\title{
Functional Design of State Estimation Based on Dispatching And Control Cloud
}

\author{
Zhengwei Jiang ${ }^{1}$, Ke Yang ${ }^{2,3}$,Lingyan Que ${ }^{1}$,Min Lu ${ }^{1}$,Yi Wang ${ }^{2,3}$, Yuchun Luo ${ }^{2,3}$ \\ 1. State Grid Zhejiang Electric Power Co.Ltd,HangZhou,China \\ 2. NARI Group Corporation (State Grid Electric Power Research Institute), NanJing, China \\ 3.NARI Technology Co. Ltd, NanJing, China \\ Jiang_zhengwei@zj.sgcc.com, Que_lingyan@zj.sgc.com,Lu_ming@zj.sgcc.com, yangke@sgepri.sgcc.com.cn,wang- \\ yi2@sgepri.sgcc.com.cn, yuoluchun@sgepri.sgcc.com.cn
}

\begin{abstract}
State estimation is widely used in Zone $I$, and provides a solid support for safe and stable operation of dispatching system and advanced application calculation. However, after the application of analysis class is extended to Zone III, the application scope is obviously increased, and the service invocation will be significantly increased. Higher requirements are also put forward for the service capability and processing capability of analysis class applications. In order to solve the problems of multi-level scheduling and hierarchical deployment, difficulties in data sharing, and insufficient support capability for multi-user and multi-demand of state estimation applications, etc. In this paper, the state estimation function and service mode based on the dispatching cloud platform are proposed, and a set of computational service flow and application mode for multi-user on-demand are described.
\end{abstract}

Keywords—state estimation; dispatching cloud; multi-user; service

\section{INTRODUCTION}

State estimation, dispatcher power flow, static security analysis and other network analysis applications are important real-time research and analysis tools for power systems [1 2] . It plays an important role in real-time dispatching and preconceived analysis of dispatch and control centers.

Network analysis application module have been widely deployed in secure zone I, it provides a strong support for the safe and stable operation of dispatching. However, it has not been effectively extended to zone III, which limits the scope of service of application analysis applications. After the analysis class application is extended to the area III, the application scope is obviously increased, and the service invocation will be significantly increased, which puts forward higher requirements for the service ability and processing ability of the analysis class application. Cloud computing technology [3] as a brand-new service model developed rapidly in recent years, has the characteristics of ultra-large-scale, virtualization, high reliability, versatility, high scalability, on-demand service, and many scholars have carried out research work on power system cloud computing platform and application computing model [4 7]. This paper mainly discusses the construction scheme and application prospect of power system cloud computing platform, the dispatching application framework of cloud computing mode, the main standards and key technologies of power system cloud computing center, heterogeneous model fusion technology, and power grid training simulation design and on-line analysis and calculation technology of whole network integration. Make use of the method to solve the challenging problem in power system computing and information processing.

This paper analyzes the requirements of network analysis application for dispatching cloud systems, and studies the key technologies of service transformation, dynamic allocation of computing resources and multi-user scenarios of network analysis application in zone III. A solution for state estimation module is proposed in the paper.

\section{DisPatching Cloud STATE Estimation OF POWER GRID MODELS AND REAL-TIME DATA STREAMS}

In the dispatching cloud mode, the cloud end deploys the unified maintenance of model data and the whole network publishing function, each application end and the source data end can receive the model data by subscription, thus realizing the unified management of the whole network model data.

The dispatching cloud state estimation model adopts a unified, standardized and standard data model for the whole network. On the one hand, it solves the drawbacks of multilevel deployment, multi-level data storage and data non-sharing in current control system; On the other hand, compared with the traditional up-splicing model which use a method by adopting equivalent load or generator at the boundary, users at all levels can quickly extract local data from cloud model data platform and external power grid plant-station data, which can effectively improves the accuracy of state estimation calculation because of the sharing of data between upper and lower levels.

A new data storage method is adopted in dispatching cloud state estimation data. The new storage method isolates the storage and management of model data and cross-section data, and adopts dynamic and static separate storage and unified access. In the framework of data dynamic and static separation storage, the model data is still stored in traditional real-time database in a relational way, and the section data is stored in a column-based memory database. Its basic storage structure is as follows in Fig.1. 


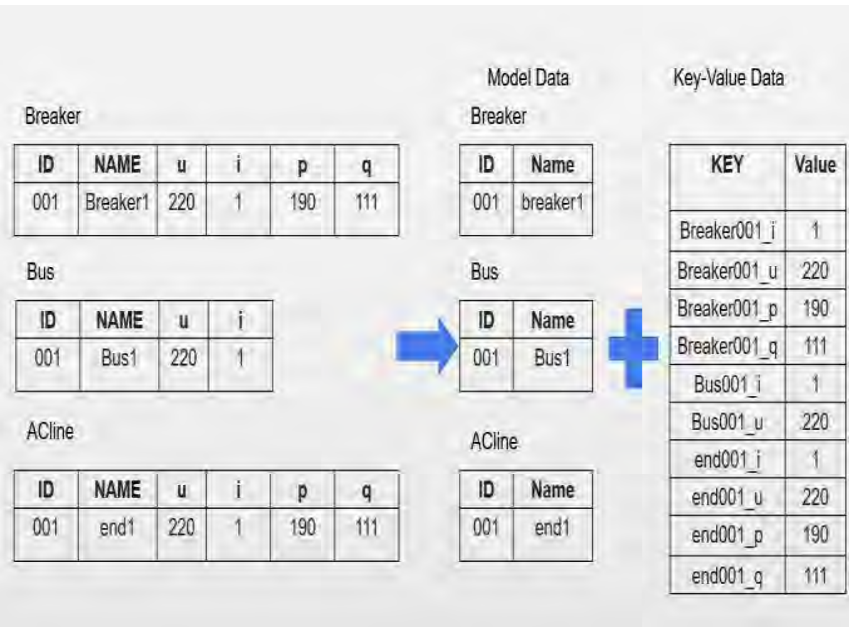

Fig. 1. Data separation structure

In the new data structure storage mode, the state estimation only needs to read the model database when the model is established, and the section data only needs to be read directly in the memory database when the model is running or triggering the calculation, which greatly improves the efficiency of reading real-time data, the dispatching cloud state estimation model and real-time data flow as follows in Fig2.

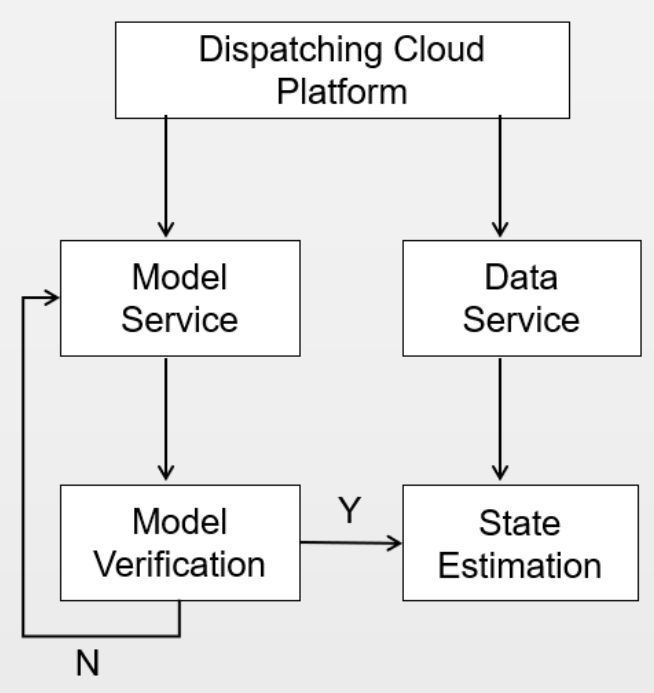

Fig. 2. State estimation process

\section{Dispatching Cloud State Estimation SERVICES ENCAPSULATION}

In cloud computing, the core issues that users are concerned about are how to obtain the computational services of state estimation and how much service capability state estimation provides.
The dispatching cloud state estimation decomposes each service, registers the corresponding service in the cloud service center, completes single service computation or multi-service composition computation.

The state estimation underlying service components adopt efficient and mature $\mathrm{C} / \mathrm{C}++$ implementations, data encapsulation uses Protocol Buffers serialization to convert to standard interfaces which publishes to the outside in the form of services .It can provides services such as model, section, file and topology according to users' needs. See TABLE 1

Model services can tailor computational models and external equivalent models from the whole network model according to the scope of the custom computational model to provide tailored models for applications.

Data section service can provide real-time and historical state estimation data section service, and other application functions can obtain the required state estimation data section only by providing the required state estimation data section time.

File service can provide model and measurement file export based on state estimation, and subsequent application or other company can calculate advanced application based on this file.

The topology display service is based on state estimation model and computational data to provide visual display of the topology map at the power plant or substation level.

The Screen display service provides real-time state estimation calculation information and responds to screen operation events for production station wiring diagram.

TABLE I. Dispatching Cloud State Estimation Services

\begin{tabular}{|l|l|}
\hline \multicolumn{1}{|c|}{ Service name } & \multicolumn{1}{|c|}{ Service description } \\
\hline Model service & $\begin{array}{l}\text { provide the tailored or whole model } \\
\text { for other application. }\end{array}$ \\
\hline Section service & $\begin{array}{l}\text { provide real-time and historical } \\
\text { state estimation data section service } \\
\text { for other application }\end{array}$ \\
\hline Topology service & $\begin{array}{l}\text { provide visual display of the } \\
\text { topology map at the area and station } \\
\text { level. }\end{array}$ \\
\hline picture service & $\begin{array}{l}\text { provides real-time state estimation } \\
\text { calculation information and } \\
\text { responds to screen operation events } \\
\text { for production station wiring } \\
\text { diagram }\end{array}$ \\
\hline
\end{tabular}

\section{Elastic EXPANSION OF Dispatching Cloud StATE ESTIMATION}

In the traditional control system, each workstation that needs to display state estimation results needs to deploy configuration files and corresponding dynamic libraries or client programs because of the $\mathrm{C} / \mathrm{S}$ mode. This presentation binds workstations to network analysis and computation instances one by one, so the number of computation / service instances is strictly limited. It is urgent to improve the management mechanism so that the computation instances can be flexibly extended. 
In the traditional dispatching and control system, because the workstation is bound to the network analysis and calculation instances one by one by configuration file, the number of calculation or service instances is strictly limited. It is urgent to improve the management mechanism so that the calculation instances can be flexibly extended.

In the dispatching cloud state estimation, users do not need any configuration, only need to select the state estimation application scenario in the browser, The resource scheduler in the control cloud platform performs unified scheduling, allocation and requests according to the resource requirements required by the user to select the application scenario, realizes the fast transition between the client and the server, and forms a point-to-point state estimation service resource with the user.

See Fig.3.

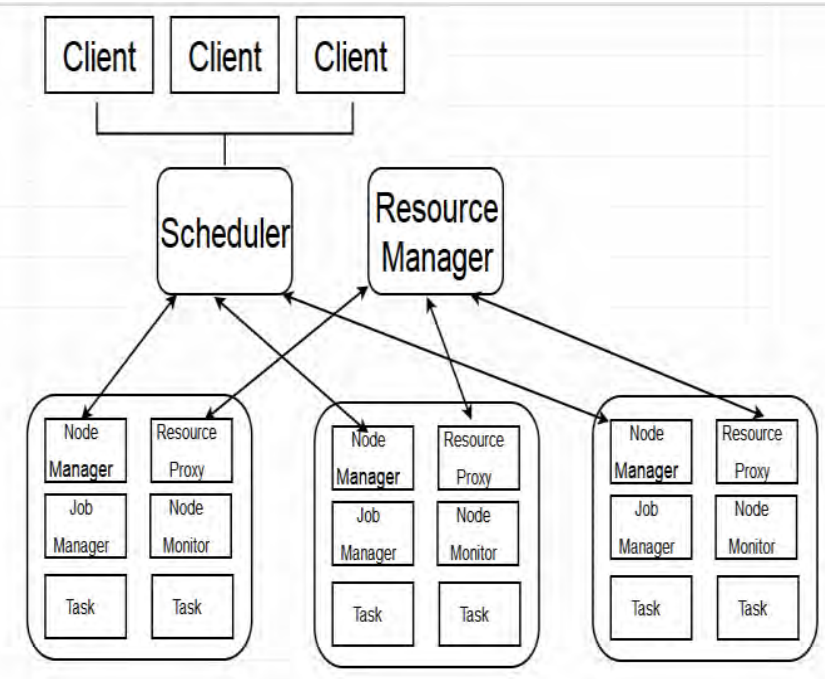

Fig. 3. Dynamic resource allocations

The client in the figure above represents the client for each state estimation, the Resource Manager in the dispatching cloud platform is the resource monitoring module, and the NodeManager and Resource Proxy constitute the server and server resource states for the state estimation.

ResourceManager is Responsible for the unified management of the resources reported by ResourceProxy and forming the resource pool of clusters. Scheduler is the task scheduler of the whole cluster. It receives the task requests submitted by Client and schedules the tasks reasonably according to the resource requirements of the task and the status of the resource pool of the cluster

This kind of B/S display and resource allocation mode, all business logic in the server side (Server) implementation, the client is easy to deploy without any configuration, greatly reducing the cost and workload of system maintenance and upgrade, enhance the scalability

\section{Dispatching Cloud State Estimation IN MUlti- USER SCENARIO}

\section{A. Multi-user result sharing}

Current state estimation applications are dispatchingoriented applications, which provide users with a unified display of results in a fixed model and measurement range. Applications at all levels of dispatchers need to be deployed, and the results are difficult to share. In fact, the application business of multi-level dispatching between provinces and provinces is similar, but the scope of model and measurement is different. The deepening of grid interconnection and the expansion of dispatching business require that the application calculation is no longer limited to their respective control scope.

This problem is solved by deploying a set of state estimation at the provincial level. Users $\log$ in to control the unified login page of the cloud portal, check the application integration home page after login for permission verification, and dynamically allocate computing resources according to state estimation when selecting the platform that needs to access the application scenario. the state estimation records the user's dispatching area, personal privileges and other information, and pushes different state estimation results. At the same time, different users are given different operation permissions according to their personal permissions, and the load balancing technology of regulating cloud platform is used to meet the multi-user simultaneous login and viewing scenario. See Fig.4

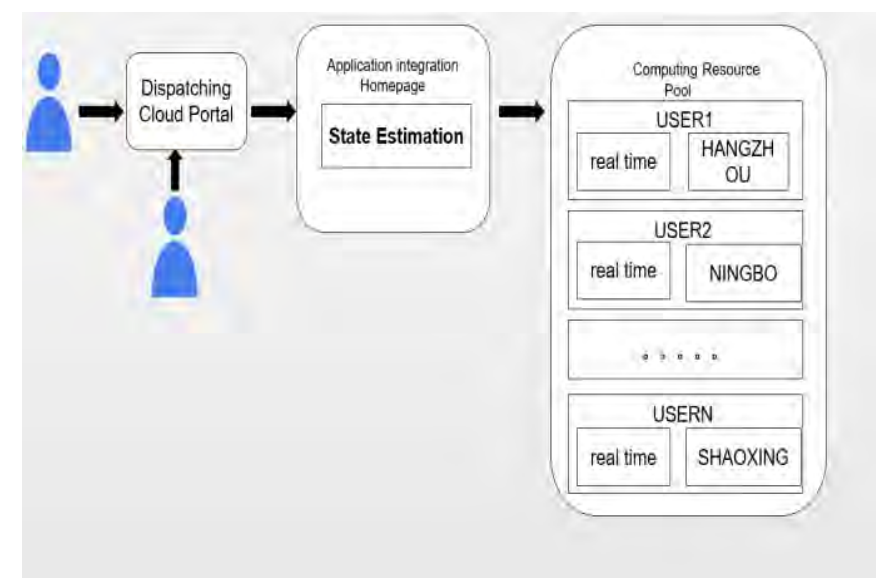

Fig. 4. State estimation multi-user scenario

\section{B. Multi-user resource isolation}

The control cloud platform provides the isolation of computing resources and the isolation of data resources for state estimation applications.

The isolation of computing resources means that the CPU and memory resources occupied by the application run are isolated from each other, thus ensuring that the task run will not preempt resources from each other and cause abnormal operation. Taking state estimation calculation as an example, each user is assigned a state estimation calculation task. If 
there is no computational resource isolation, a computing task crashes due to memory leaks and other exceptions, affecting the normal use of other users. Computing resource isolation will isolate the abnormal tasks and ensure the stability of other tasks.

Data resource isolation means that private data used in state estimation can be isolated from each other, and the same functions used by different users have independent data storage in their running resource space, and their operation and analysis results will not affect each other. Data resources of state estimation can be publicized through its data services, thus data sharing can be accomplished.

Data isolation flow chart see Fig.5

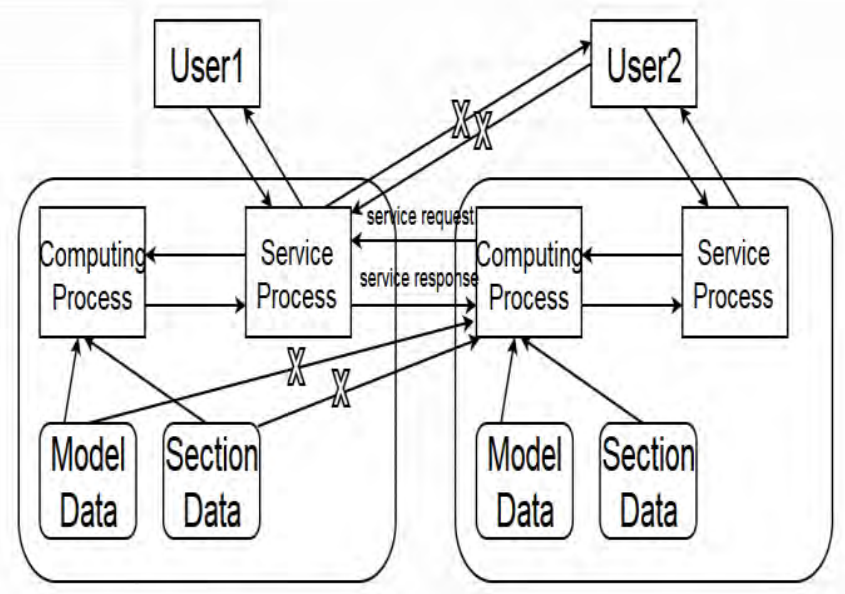

Fig. 5. Data isolation

\section{TyPiCAl CALCUlation OF STATE Estimation}

The technical scheme of state estimation based on dispatching cloud is described as follows: user logins to cloud center, cloud center distributes computing service, computing environment and computing data for users, obtains grid operation data and grid model needed by users from model data cloud platform through data service and model service respectively, and verifies the model and data. The data realtime library and model real-time library are written respectively after successful inspection, so State estimation can get corresponding models and real-time data information.

The user carries on the whole network state estimation computation, the system then pushes the corresponding state estimation result according to the user information.

Zhejiang Province dispatching cloud integrates the model and data of provincial and municipal dispatch center coverage voltage levels from $35 \mathrm{kV}$ to $1000 \mathrm{kV}$, and develops the demonstration application of the whole network state estimation service framework. Based on the public service of cloud platform, the user calls the application service and completes the model checking and state estimation calculation in local area. Greatly increased the deployment time of the prefecture level area, and improved the qualified rate of the whole network state estimation.
The comparison between traditional state estimation and regulation cloud state estimation is shown in Table 2.

TABLE II. COMPARISON OF STATE ESTIMATION RESULTS

\begin{tabular}{|c|c|c|}
\hline \multicolumn{1}{|c|}{ method } & $\begin{array}{c}\text { qualification } \\
\text { rate }\end{array}$ & $\begin{array}{c}\text { computing } \\
\text { time }\end{array}$ \\
\hline $\begin{array}{l}\text { Traditional state } \\
\text { estimation }\end{array}$ & $90.56 \%$ & $10.23 \mathrm{~s}$ \\
\hline $\begin{array}{l}\text { Dispatching cloud } \\
\text { state estimation }\end{array}$ & $97.45 \%$ & $4.78 \mathrm{~s}$ \\
\hline
\end{tabular}

\section{CONCLUSION}

State estimation application service architecture based on dispatching cloud has changed the tradition that application software needs to be deployed and used hierarchically on the upper and lower scheduling platforms, and realized the separation of computing program and computing data.

A set of service multi-user on-demand usage mode is established. Dispatching cloud state estimation based on unified data structure for computing data cloud sharing, which improves the accuracy and practicability of state estimation in provincial and municipal integration applications. It also provide data support for dispatcher power flow, static security analysis, DTS simulation and big data analysis applications.

Currently, dispatching cloud state estimation implements man-machine display, data isolation and other technologies. In the future, state estimation model and data service mechanism need to be further improved, and in-depth study on data interaction is needed.

\section{REFERENCES}

[1] Peng Qian,HuGuoxin,Zhang Li. “A load flow algorithm based on Gauss algorithm and fast decoupling algorithm".Power System Technology, vol 33,pp.53-56,2009.

[2] WangMiao,Chang Naichao,Liu Jinbo,et al. "A multi-index comprehensive evaluation method of state estimation", Automation of Electric Power Systems,vol 39,pp.94-98,2015

[3] Zhao Junhua,Wen Fushuan,Xue Yusheng,et al.Cloud computing implementing an essential computing platform for future power systems".Automation of Electric Power Systems, vol 34,issue 15,pp.1-8,2010.

[4] CaoYang,Gao Zhiyuan,Yang Shengchun,etal. "Application of cloud computing in power dispatching systems".Electric Power,vol 45,issule 6,pp.14-17,2012

[5] Wang Shouxiang,Zhang Weitao,Ge Leijiao."Unified model cloud and application service system for smart distribution network".Electric PowerAutomationEquipment,vol 35, issule 2,pp.49:54,2015.

[6] Pu Tianjiao,Wang Xiaohui,Li Zhihong,et al."Architecture and key technology of power grid dispatch training simulation based on cloud computing".Power System Technology,vol 40,pp.2533-2540,2016

[7] Lin Jinghuai."Cloud computing based system design for power grid dispatching and control training simulation".Automation of Electric Power Systems, vol 41,pp,164-170,2017 